\title{
Impact of hypocaloric dietary intervention on ovulation in obese women with PCOS
}

\author{
Brittany Y Jarrett and Marla E Lujan \\ Human Metabolic Research Unit, Division of Nutritional Sciences, Cornell University, Ithaca, New York, USA \\ Correspondence should be addressed to M E Lujan; Email: marla.lujan@cornell.edu
}

\begin{abstract}
Polycystic ovary syndrome (PCOS) is a common cause of ovulatory dysfunction affecting women of reproductive age. Obesity and insulin resistance are thought to potentiate disruptions in antral follicle development that result in chronic anovulation, and as such, have become important therapeutic targets of dietary interventions aimed at weight loss. Caloric restriction has been shown to promote sporadic ovulation in obese women with PCOS, but improvements have occurred across a wide range of patients and little has been garnered about the factors that distinguish responders from non-responders. Further, few studies have evaluated the likelihood for modest weight loss to restore normal ovulatory cyclicity in PCOS. Consensus regarding the impact of dietary intervention on ovulation has been limited by variability in the measures used to characterize and report ovulatory status across studies. In response, this review provides an assessment of the evidence surrounding the effectiveness of hypocaloric dietary intervention to normalize ovulatory function in PCOS. The impact of physiological vs methodological factors on the evaluation of ovulatory status is discussed, and recommendations to strengthen future studies in this area are provided. Ultimately, further research is needed to understand the optimal dietary or lifestyle approaches that promote ovulation and sustained improvements in reproductive function in PCOS.

Reproduction (2017) 153 R15-R27
\end{abstract}

\section{Introduction}

Polycystic ovary syndrome (PCOS) is the leading cause of anovulatory infertility and has broad implications for the reproductive and metabolic health of women across the lifespan (Fauser et al. 2012). The reproductive phenotype manifests as anovulation, menstrual cycle irregularity and hyperandrogenism and reflects defects at multiple levels of the hypothalamic-pituitary-ovarian axis (Jonard \& Dewailly 2004, Franks et al. 2008). The hypersecretion of luteinizing hormone by the pituitary and overproduction of androgens by the ovaries interact to impair ovarian antral follicle development (Jonard \& Dewailly 2004, Franks et al. 2008). Abnormal folliculogenesis in PCOS is characterized by an accumulation of small follicles, inhibition of terminal follicular growth (called follicle 'arrest') and failure of the mechanisms driving morphologic selection and ovulation (Jonard \& Dewailly 2004, Franks et al. 2008). Obesity is intimately linked with the pathogenesis of anovulation in PCOS (Rojas et al. 2014) (Fig. 1). Excess weight and visceral adiposity promote the development of insulin resistance and compensatory hyperinsulinemia (Diamanti-Kandarakis \& Dunaif 2012), which are posited to exacerbate disruptions in antral follicle development (Jonard \& Dewailly 2004, Franks et al. 2008) and worsen the severity of the reproductive phenotype (Lim et al. 2013). Therefore, therapies that attenuate obesity and insulin resistance hold promise to normalize anovulation and hyperandrogenism in PCOS (Fig. 1).

Currently, dietary interventions involving caloric restriction are recommended to combat both reproductive and metabolic abnormalities in overweight and obese women with PCOS (Moran et al. 2009, 2013, Fauser et al. 2012, Legro et al. 2013). Modest reductions in energy intake (500-1000 kcal/day) and weight (5-10\%) have been shown to normalize gonadotropin secretion (Bützow et al. 2000), reduce clinical and biochemical hyperandrogenism and improve insulin sensitivity in this population (Moran et al. 2009, 2011). Likewise, randomized and non-randomized trials have documented an increased frequency of spontaneous ovulation, menses and pregnancy with weight loss (Moran et al. 2009). These findings are thought to reflect a recovery of the hormonal features that underpin follicular excess and 'arrest' in PCOS (Moran et al. 2011) (Fig. 1). However, there are several challenges to understanding the actual effectiveness of weight loss to stimulate ovulation or restore normal ovulatory function in overweight and obese patients. Despite evidence of ovulation after dietary interventions, variability in the measures used to report endpoints across studies 


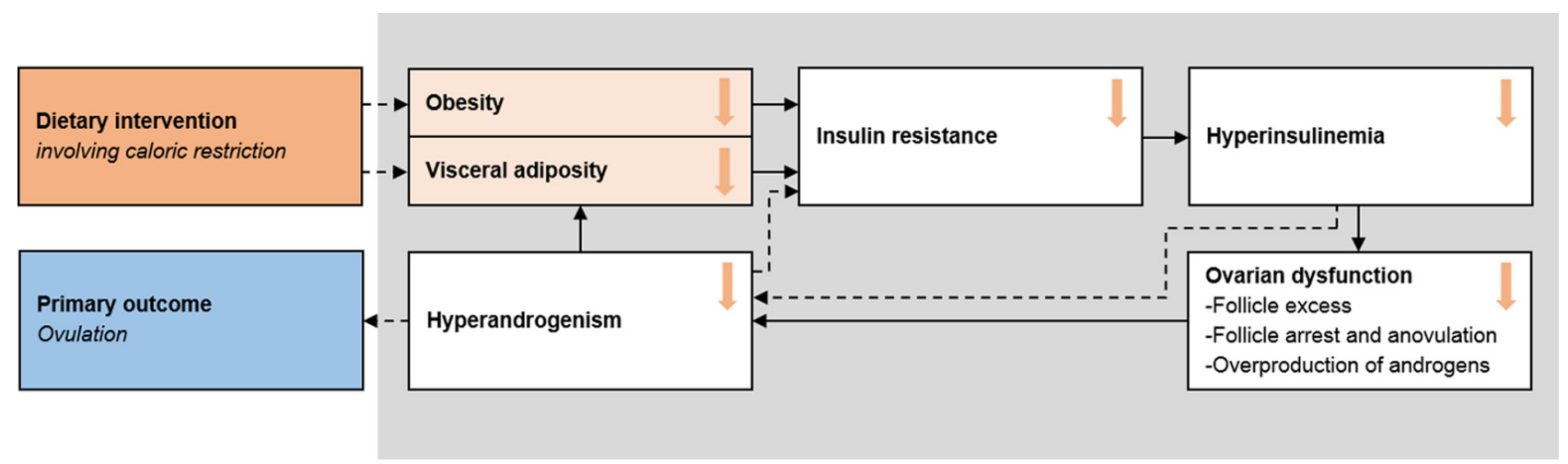

Figure 1 Proposed mechanism by which hypocaloric dietary intervention promotes ovulation in obese women with PCOS. As shown in the gray inset, excess weight and visceral adiposity promote the development of insulin resistance and compensatory hyperinsulinemia. Both are posited to exacerbate disruptions in antral follicle development, through a reciprocal relationship with hyperandrogenism. Insulin contributes to systemic hyperandrogenism by stimulating the production of androgens by the ovaries and inhibiting the synthesis of sex hormone-binding globulin by the liver. These direct (solid line) and indirect actions (dotted line) of insulin result in elevated circulating concentrations of bioavailable androgens. Androgens may promote preferential deposition of adipose tissue in the abdomen and can exacerbate insulin resistance through obesogenic mechanisms (solid line) or independently (solid line) by modifying insulin action in classic target tissues. As dietary interventions attenuate obesity and insulin resistance (orange boxes and arrows), they hold promise to treat anovulation and hyperandrogenism in PCOS.

has prevented systematic assessments of the impact of weight loss on reproductive outcomes (Moran et al. 2011). Further, improvements in ovulation have been noted over a wide range of women (Moran et al. 2009, Legro et al. 2013); yet, little work has been done to understand the factors that account for the variability in the ovulatory response to caloric restriction.

To that end, the purpose of this review was to assess the evidence surrounding the effectiveness of hypocaloric dietary intervention to normalize ovulatory function in PCOS. The occurrence of and factors associated with ovulation in response to modest weight loss are described. Particular consideration is given to inconsistencies in the methods used to characterize ovulatory status across studies. The impact of physiological and methodological factors on the evaluation of ovulatory potential prior to dietary intervention is also discussed.

\section{Literature search and selection criteria}

PubMed, CINAHL and the Cochrane Central Register of Controlled Trials were used to identify relevant studies published between January 1990 and March 2016. Bibliographies of related systematic or narrative review articles were also screened to identify additional studies. The search included a combination of keywords relevant to PCOS, dietary or lifestyle intervention, and ovulation and menstrual cyclicity. The Population, Intervention, Comparison, Outcome (PICO) framework was used to define the inclusion and exclusion criteria for studies a priori. Briefly, studies included in this review were limited to original research articles in which (1) the patient population comprised only overweight or obese women with PCOS; (2) the dietary intervention involved reductions in energy intake that were intended to promote weight loss and (3) the primary or secondary outcome of interest was ovulation. Only articles published in English were included. Overweight or obesity was defined as a BMI of $25.0-29.9 \mathrm{~kg} / \mathrm{m}^{2}$ (overweight) or $\geq 30 \mathrm{~kg} / \mathrm{m}^{2}$ (obesity) (Jensen et al. 2014). PCOS was defined according to the National Institutes of Health (NIH) (Zawadzki \& Dunaif 1992) or Rotterdam criteria (Rotterdam ESHRE/ASRM-Sponsored PCOS Consensus Workshop Group 2004a,b). Randomized controlled trials and non-randomized intervention studies were considered. Trials that incorporated either supervised or unsupervised physical activity with caloric restriction were included. By contrast, studies with combined dietary and pharmaceutical interventions (i.e. metformin or clomiphene citrate) were excluded, unless the pharmaceutical therapy served as a comparison to changes in energy intake alone. The title and abstract of every record retrieved by this search strategy was checked to ensure that it aligned with the established inclusion criteria. Relevant articles were downloaded for full-text review. Data on general characteristics of the study, patient population, diagnosis of PCOS, inclusion and exclusion criteria, intervention design, measurement of ovulation and outcomes related to ovulatory function were extracted.

\section{Characteristics of the studies included for review}

The search returned 4046 records, including ones that were identified through electronic databases $(n=3319)$ and bibliographies of other reviews $(n=727)$. Duplicates found using multiple databases, keywords and sources were removed $(n=3234)$. All remaining records $(n=812)$ were evaluated in the context of the PICO framework, and 780 were excluded based on 


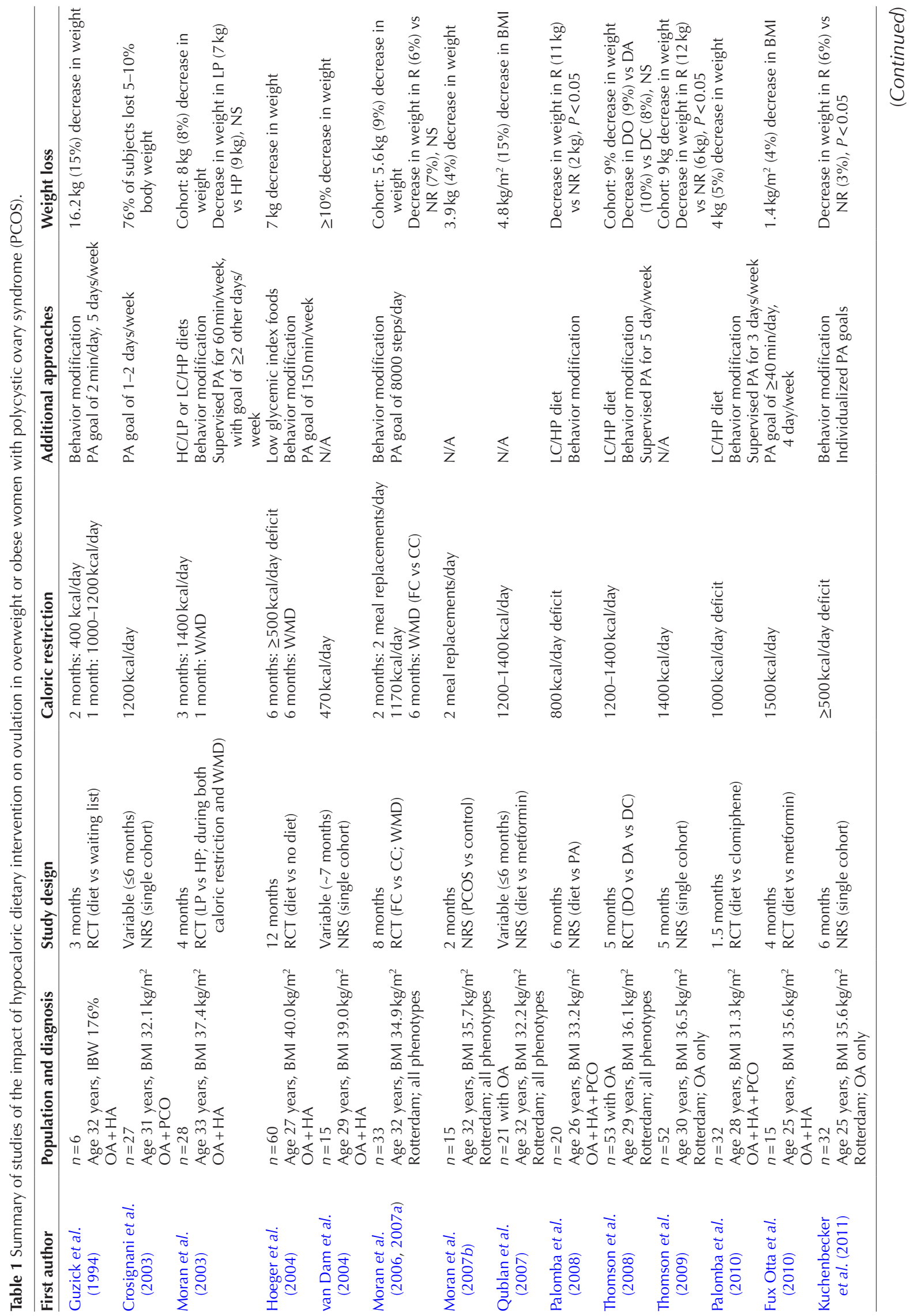




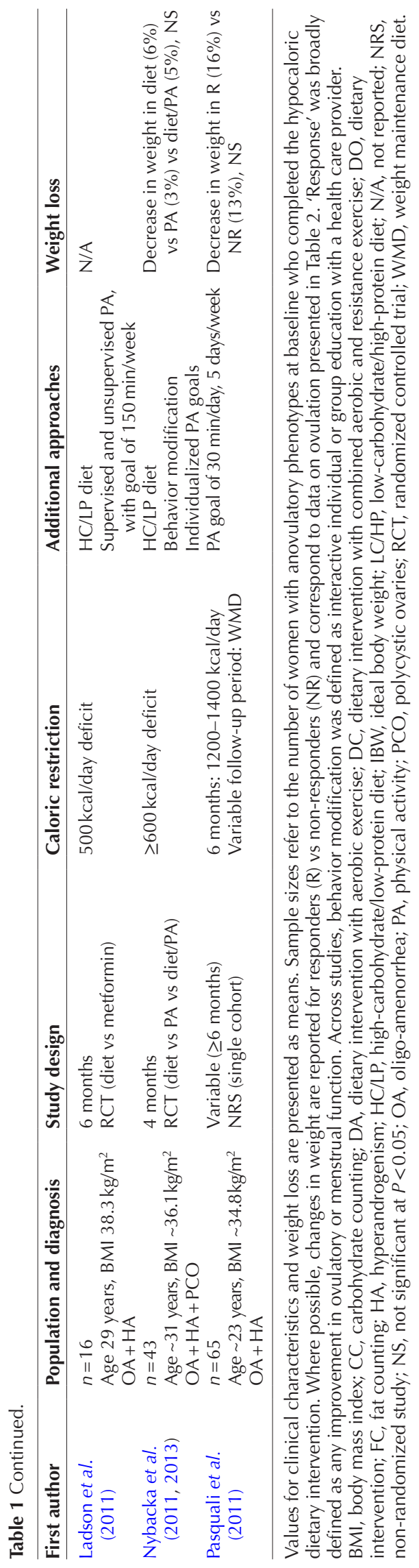

the information provided in the title and/or abstract. Thirty-two original research articles were selected for full-text review. Of these, 13 were excluded due to the inclusion of women without PCOS $(n=2)$, use of inappropriate diagnostic criteria $(n=1)$, use of eucaloric dietary interventions $(n=3)$ or the absence of outcome data on ovulation $(n=7)$. Ultimately, 19 articles from 17 different studies were included for review. Two of the articles constituted secondary analyses (Moran et al. 2007a, Nybacka et al. 2013) of data collected during a previous dietary intervention (Moran et al. 2006, Nybacka et al. 2011).

Relevant study characteristics are summarized in Table 1. At enrollment, the mean age of participants ranged from 23 to 33 years, and mean BMI was within the obese category (Table 1 , Column 2). All of the dietary interventions were aimed at modest weight loss, primarily through short-term reductions in energy intake. In general, women were prescribed a caloric deficit of at least $500 \mathrm{kcal}$ per day and encouraged to restrict energy intake to between 1000 and $1400 \mathrm{kcal}$ per day. Two studies used very low-calorie diets (i.e. total energy intake $<500 \mathrm{kcal}$ per day) (Guzick et al. 1994, van Dam et al. 2004) (Table 1, Column 4). Despite being hypocaloric, the dietary interventions met national standards for carbohydrates $(45-65 \%$ of calories), protein (10-35\% of calories) and fat (20-35\% of calories) (Institute of Medicine 2005). Participants were largely required to purchase and prepare their own meals and snacks; four studies provided partial or complete meal replacements to aid in caloric restriction (Guzick et al. 1994, van Dam et al. 2004, Moran et al. 2006, 2007b). Multifactorial approaches to enhance the target energy deficit and degree of weight loss were also common. Some studies modified macronutrient composition, recommended a specific frequency or intensity of physical activity and/or delivered behavior modification therapy through individual or group education sessions (Table 1, Column 5). Fifteen of the 17 studies were conducted over a period of three months or longer, wherein the duration of active weight loss ranged from six weeks (Palomba et al. 2010) to seven months (van Dam et al. 2004) (Table 1, Columns 3-4). At the end of the dietary interventions, mean reductions in body weight ranged from 3\% (Kuchenbecker et al. 2011, Nybacka et al. 2011) to 16\% (Pasquali et al. 2011) (Table 1, Column 6).

Improvements in ovulation were defined in one of two ways across studies: (1) the occurrence of one or two ('sporadic') ovulations or (2) the resumption of regular ('monthly') ovulatory cycles. Data were reported as the proportion of women with either ovulatory response or as the number of ovulatory menstrual cycles detected during the dietary intervention. Overall, most of the results on improved ovulatory function were presented as the number of women who experienced sporadic ovulation with weight loss. Only two of the studies 
assessed the occurrence of regular ovulatory cycles (Crosignani et al. 2003, Thomson et al. 2008), and none compared the likelihood for dietary intervention to stimulate a single ovulation vs restore normal ovulatory function. Similarly, three studies published data on the number of ovulatory menstrual cycles detected per woman or treatment group, but did not provide any additional information with which to characterize ovulatory cyclicity (Hoeger et al. 2004, Palomba et al. 2008, Ladson et al. 2011).

\section{Dichotomization of women as responders and non-responders to dietary intervention}

Based on these definitions, the ovulatory response to hypocaloric dietary intervention was decidedly variable between individuals and across studies (Table 2, Columns 6-7). Both sporadic and regular ovulations were detected in a wide range of participants with either self-reported or confirmed menstrual cycle irregularity at baseline. Thirteen to $85 \%$ of women experienced sporadic ovulation with weight loss and no more than $55 \%$ resumed regular ovulatory cycles (Table 2 , Columns 6-7). On average, fewer than three ovulations were detected per woman during a six-month dietary intervention (Hoeger et al. 2004, Palomba et al. 2008, Ladson et al. 2011). Together, these data imply continued cycle irregularity with ovulation occurring at least two months apart.

In all studies, a subset of women remained anovulatory despite being compliant with the dietary intervention (as judged by weight loss) (Table 2, Columns 6-7). Hence, women could be dichotomized as 'responders' and 'non-responders' to weight loss. These results could be interpreted to mean that dietary intervention is not a universal solution for anovulation in PCOS. Yet, it is prudent to consider that variability in the ovulatory response may stem from (a) inconsistencies in the measurement of ovulation across studies, (b) heterogeneity in the clinical presentation of PCOS or (c) the degree of change in salient endocrine or metabolic features during the dietary intervention. The potential impact of each of these factors on the evaluation of the ovulatory response to weight loss is addressed in the sections that follow.

\section{Inconsistencies in the methods used to measure ovulatory status}

In line with previous reports (Moran et al. 2011), the methods used to detect ovulation and characterize any reinstatement of ovulatory cyclicity were inconsistent across studies (Table 2, Columns 1-3). As shown in Table 2 (Column 2), the primary markers of ovulation were elevated serum concentrations of progesterone or increased urinary excretion of pregnanediol

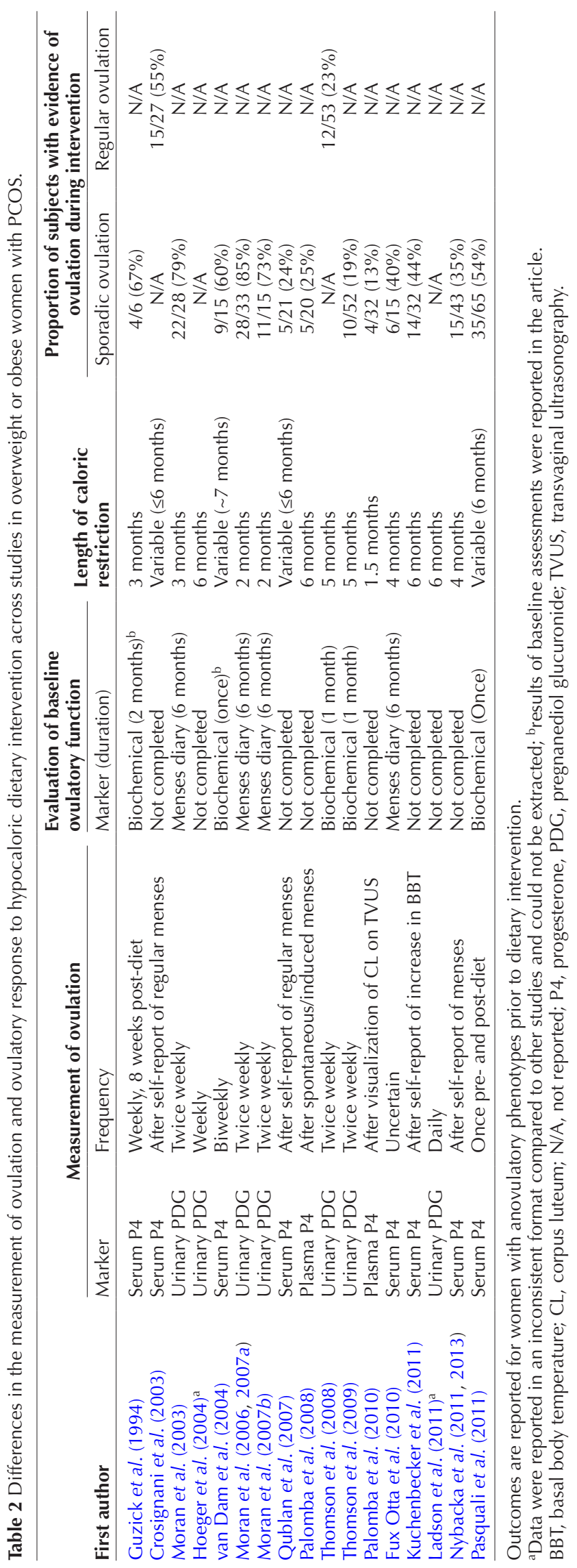

Reproduction (2017) 153 R15-R27 
3-glucuronide (PDG). These measurements were consistently taken during the mid-luteal phase of the menstrual cycle. Several studies also evaluated the urinary estrogens in conjunction with PDG (Moran et al. 2003) or considered pregnancy to be sufficient evidence of ovulation in lieu of serum progesterone (Crosignani et al. 2003, Kuchenbecker et al. 2011).

In both clinical practice and research, a number of techniques are used to confirm the occurrence and timing of ovulation. The gold standard method involves the direct observation of follicular growth and rupture by high-resolution transvaginal ultrasonography (Baerwald et al. 2012). If this approach is not feasible, then other indirect, but objective, methods may be employed to detect ovulation. Such methods include the measurement of pituitary or ovarian hormones in the blood, urine or saliva, and the assessment of clinical symptoms including menstrual cycle length or basal body temperature (BBT) (Campbell \& Rockett 2006, Lynch et al. 2006). In line with these standards, the studies were justified in their common use of serum progesterone or urinary PDG (Table 2, Column 2); both have been validated as reliable and interchangeable markers of ovulation in women with regular menstrual cycles (Roos et al. 2015). A sustained elevation in either biochemical marker is considered sufficient evidence of luteal activity and can be detected throughout the luteal phase to the end of the cycle (Roos et al. 2015).

That being said, the frequency at which these biochemical markers were measured differed substantially across studies. Approximately half of the studies $(n=8)$ used an intermittent sampling design irrespective of the stage of the menstrual cycle. Assessments were performed daily (Ladson et al. 2011), twice weekly (Moran et al. 2003, 2006, 2007b, Thomson et al. 2008, 2009), weekly (Hoeger et al. 2004) or biweekly (van Dam et al. 2004) to detect the ovulation during the dietary intervention (Table 2, Column 3). By contrast, six studies collected a single blood sample in the mid-luteal phase based on morphologic or clinical markers (Crosignani et al. 2003, Qublan et al. 2007, Palomba et al. 2008, 2010, Kuchenbecker et al. 2011, Nybacka et al. 2011). Of these, only one used direct methods to monitor follicle growth and determine ovulation (Palomba et al. 2010). Namely, Palomba and coworkers performed transvaginal ultrasonography at baseline, every four days until visualization of a dominant follicle and then daily to follicular collapse. Ovulation was subsequently confirmed with plasma progesterone levels (Palomba et al. 2010). The other five studies relied on participant self-report of recent spontaneous menses (Palomba et al. 2008, Nybacka et al. 2011), resumption of regular menses (Crosignani et al. 2003, Qublan et al. 2007) or increase in BBT (Kuchenbecker et al. 2011) (Table 2, Column 3). When one of these events occurred, the investigators scheduled a blood draw for serum progesterone based on an estimation of time to the next luteal phase (Crosignani et al. 2003, Qublan et al. 2007, Palomba et al. 2008, Kuchenbecker et al. 2011, Nybacka et al. 2011). Finally, of the remaining three studies, one did not provide adequate information with which to judge the frequency of measurements (Fux Otta et al. 2010) and two did not assess ovulation until after the intervention had ended (Guzick et al. 1994, Pasquali et al. 2011) (Table 2, Column 3).

It is probable that the studies with intermittent sampling designs were better positioned to capture ovulatory status than the studies that relied on a single biochemical measurement or self-reported clinical data. The occurrence of ovulation is best ascertained with an intermittent sampling design that allows for hormone data to be collected either daily, every-other-day, twice weekly or weekly ( $\mathrm{O}^{\prime}$ Connor et al. 2006). If one of these approaches is not feasible, a single measurement of serum progesterone or urinary PDG in the midluteal phase can also be effective to determine whether ovulation has occurred (Leiva et al. 2015). However, the reliability of a single measurement to detect ovulation during long or unpredictable menstrual cycles, capture peak progesterone concentrations or judge luteal phase sufficiency is uncertain. Similar to biochemical methods, prospective menstrual diaries and BBT records have been shown to provide reliable estimates of menstrual regularity (Creinin et al. 2004) and the presence of ovulation in healthy women (Campbell \& Rockett 2006, Lynch et al. 2006). Yet, variability in consecutive cycle lengths is common (Creinin et al. 2004) and ovulation is not an inevitable event during spontaneous menstrual cycles (Prior et al. 2015). Infertile patients also demonstrate abnormal fluctuations in BBT during ovulatory cycles, leading to errors in the interpretation of temperature charts and prediction of ovulation (Lenton et al. 1977). Given these challenges with self-reported clinical data, the choice to schedule blood draws during the predicted luteal phase of the next menstrual cycle implies that the first ovulation was never biochemically confirmed (Crosignani et al. 2003, Qublan et al. 2007, Palomba et al. 2008, Kuchenbecker et al. 2011, Nybacka et al. 2011). Such approaches may have resulted in a poor estimation of the number of ovulatory cycles, and consequently, a reduced likelihood to accurately report the ovulatory response to weight loss.

There was also marked variability in the methods used to define biochemical evidence of ovulation across studies. In the interventions that assessed serum progesterone (Table 2, Column 2), ovulatory thresholds were always reported and ranged from $\geq 4 \mathrm{ng} / \mathrm{mL}$ (13 nmol/L) (Fux Otta et al. 2010) to $\geq 10 \mathrm{ng} / \mathrm{mL}$ (32 nmol/L) (Qublan et al. 2007, Palomba et al. 2008, 2010). It was difficult to discern whether these thresholds were internally validated or reflected the limits of detection of the various assays used. Two of the studies implemented a commercial radioimmunoassay 
(van Dam et al. 2004, Qublan et al. 2007), but the others did not describe the methods employed to measure serum progesterone. By contrast, normative values for PDG were not provided by any of the studies that used the marker, and the increase in mid-luteal excretion was qualitatively assessed (Moran et al. 2003, 2006, 2007b, Hoeger et al. 2004, Thomson et al. 2008, 2009, Nybacka et al. 2011).

Accordingly, the interpretation of ovulatory status may have been limited by the use of inappropriate thresholds for progesterone and PDG to confirm ovulation. In general, a serum progesterone concentration of $3.9 \mathrm{ng} / \mathrm{mL}(12.5 \mathrm{nmol} / \mathrm{L})$ or higher is thought to be presumptive evidence of an ovulatory cycle using commonly available commercial assays (Wathen et al. 1984, Leiva et al. 2015). However, all the studies identified thresholds for progesterone that were above this concentration (Guzick et al. 1994, Crosignani et al. 2003, van Dam et al. 2004, Qublan et al. 2007, Palomba et al. 2008, 2010, Fux Otta et al. 2010, Kuchenbecker et al. 2011, Pasquali et al. 2011). This is interesting, considering that the studies that relied on the highest thresholds $(\geq 32 \mathrm{nmol} / \mathrm{L})$ were also the ones to report the smallest proportion of responders to the dietary intervention (Qublan et al. 2007, Palomba et al. 2008, 2010) (Table 2, Column 6). In addition, there is emerging evidence to suggest that luteal phase dynamics of progesterone are altered in obesity (Rochester et al. 2009) and PCOS (Joseph-Horne et al. 2002). Lower urinary excretion and a delayed ovulatory rise in PDG have been noted in obese women with regular menstrual cycles (Rochester et al. 2009), and lower luteal concentrations of progesterone have been documented in women with ovulatory PCOS compared to healthy controls (Joseph-Horne et al. 2002). These findings have implications for the detection of spontaneous ovulation in obese anovulatory patients and suggest that alternative thresholds may be needed to fully capture ovulatory status in PCOS.

\section{Heterogeneity in the clinical presentation of PCOS}

The dichotomization of the ovulatory response to hypocaloric dietary intervention likely reflects the heterogeneous nature of PCOS. PCOS exists on a spectrum, and the diagnosis encompasses a broad range of severity of reproductive and metabolic abnormalities (Diamanti-Kandarakis \& Dunaif 2012, Fauser et al. 2012). Such differences may impart a variable potential between patients for weight loss to stimulate ovulation. To that end, there is utility in identifying the endocrine or metabolic characteristics that distinguish responders from non-responders prior to dietary intervention. Nine of the studies evaluated baseline clinical predictors of improvements in reproductive function (Moran et al. 2003, 2007a, van Dam et al. 2004, Thomson et al. 2008, 2009, Kuchenbecker et al. 2011, Nybacka et al. 2011, 2013, Pasquali et al. 2011).

Response was defined in one of two ways across studies: (1) evidence of sporadic ovulation or (2) improved menstrual cyclicity after dietary intervention. None of the studies assessed predictors of the transition to normal ovulatory function. Improvements in menstrual cyclicity were broadly characterized as a decrease in menstrual cycle irregularity, shift from irregular to regular menstrual cycles or shift from anovulatory to ovulatory cycles (Moran et al. 2003, 2007a, Thomson et al. 2008, 2009, Nybacka et al. 2011, 2013). Increased cycle regularity was not necessarily accompanied by improvements in ovulation and a shift from anovulatory to ovulatory cycles appeared to reflect evidence of sporadic, rather than regular, ovulation (Moran et al. 2003, 2007a, Thomson et al. 2009). In addition, one study evaluated women with partial or complete recovery from PCOS and identified baseline features associated with the collective normalization of hirsutism, ovulation and menstrual cyclicity (Pasquali et al. 2011).

As shown in Table 3, six of the nine studies identified significant clinical predictors of improved reproductive outcomes after dietary intervention (van Dam et al. 2004, Moran et al. 2007a, Thomson et al. 2009, Nybacka et al. 2011, 2013, Pasquali et al. 2011). The baseline characteristics that emerged were largely markers of androgen excess and adiposity. Overall, women with lower circulating concentrations of sex hormone-binding globulin (van Dam et al. 2004), testosterone (Nybacka et al. 2011), androstenedione (Pasquali et al. 2011) and anti-Müllerian hormone

Table 3 Baseline endocrine and metabolic characteristics linked to improved reproductive outcomes after hypocaloric dietary intervention in overweight or obese women with PCOS.

\begin{tabular}{lll}
\hline Outcome & Baseline characteristic of responders $^{\mathbf{a}}$ & Study $^{-}$ \\
\hline Sporadic ovulation & None & Kuchenbecker et al. (2011), Nybacka et al. (2013) \\
& Higher SHBG & van Dam et al. (2004) \\
Regular (monthly) ovulation & None & None \\
Improved menstrual cyclicity & None & Moran et al. (2003), Thomson et al. (2008) \\
& Lower AMH & Moran et al. (2007a), Thomson et al. (2009), Nybacka et al. (2013) \\
Recovery from PCOS & Lower testosterone & Nybacka et al. (2011) \\
& Lower androstenedione & Pasquali et al. (2011) \\
& Lower waist circumference and WHR & Pasquali et al. (2011) \\
\hline
\end{tabular}

${ }^{\mathrm{a}}$ vs non-responders.

AMH, anti-Müllerian hormone; SHBG, sex hormone-binding globulin; WHR, waist-to-hips ratio. 
(AMH) (Moran et al. 2007a, Thomson et al. 2009, Nybacka et al. 2013) were more likely to experience sporadic ovulation and improved menstrual cyclicity during dietary intervention. Lower waist circumference and waist-to-hip ratio were also predictive of recovery from the syndrome (Pasquali et al. 2011). Taken together, these findings suggest that obese women with milder ovarian dysfunction at baseline may be more likely to experience reproductive benefit from hypocaloric dietary intervention (Table 3).

That being said, only three studies directly focused on clinical predictors of ovulation, and of these, two could not identify baseline differences between responders and non-responders (Kuchenbecker et al. 2011, Nybacka et al. 2013). Despite their associations with improved menstrual cyclicity, neither androgens nor AMH predicted ovulation after dietary intervention (Kuchenbecker et al. 2011, Nybacka et al. 2013) (Table 3). Inconsistencies in the ability to identify baseline predictors of ovulatory response may have been influenced by two important factors.

First, 'response' was broadly defined as an improvement in ovulation or menstrual cyclicity compared to baseline. However, very few studies assessed baseline ovulatory status in their participants (Table 2, Column 4). Among the studies that did, serum progesterone or urinary PDG were measured at a single time point (van Dam et al. 2004, Pasquali et al. 2011) or on a serial basis for up to two months prior to intervention (Guzick et al. 1994, Thomson et al. 2008, 2009). Alternatively, some participants were asked to keep menstrual diaries for one to six months (Moran et al. 2003, 2006, 2007b, Thomson et al. 2008, 2009, Fux Otta et al. 2010). Although these direct and indirect markers of ovulation were likely used as a reference for improvement, only two of the studies actually published the data that were collected (Guzick et al. 1994, van Dam et al. 2004) (Table 2, Column 4). As a result, it was difficult to confirm that the reported changes constituted an improvement compared to baseline and a missed opportunity was noted to characterize the variability in the degree of ovulatory response among women. The latter points to some uncertainty that a single baseline characteristic could predict a broad spectrum of reproductive improvements after dietary intervention.

Second, the use of different diagnostic criteria to define study populations resulted in the assessment of ovulatory response across multiple phenotypes (Table 1 , Column 2). The most commonly accepted criteria for the diagnosis of PCOS (2003 Rotterdam Criteria) identify four distinct clinical phenotypes: (1) Frank (oligomenorrhea, hyperandrogenism and polycystic ovaries), (2) NonPCO (oligomenorrhea, hyperandrogenism and normal ovaries), (3) ovulatory (regular menses, hyperandrogenism and polycystic ovaries) and (4) normoandrogenic or 'mild' (oligomenorrhea, normal androgen status and polycystic ovaries) (Rotterdam ESHRE/ASRM-Sponsored
PCOS Consensus Workshop Group 2004a,b). There is substantial evidence that the severest variants of the condition are Frank and Non-PCO PCOS (phenotypes that are also recognized by the $1990 \mathrm{NIH}$ Criteria) (Zawadzki \& Dunaif 1992, Fauser et al. 2012). Women with combined oligomenorrhea and hyperandrogenism have the most profound disturbances in gonadotropin dynamics, ovarian androgen production and insulin sensitivity, independent of obesity (Carmina et al. 2005, Dewailly et al. 2006, Panidis et al. 2012). By contrast, the other phenotypes seem to represent milder variants of the condition. Women with ovulatory and normoandrogenic PCOS have endocrine disturbances that are intermediate to Frank PCOS and healthy controls (Carmina et al. 2005, Dewailly et al. 2006, Panidis et al. 2012), and the presence of metabolic abnormalities seems to depend on the degree of abdominal adiposity (Moran \& Teede 2009). Collectively, these differences suggest that variable improvements in endocrine and metabolic abnormalities may be needed to restore ovulatory cyclicity across phenotypes.

PCOS was diagnosed according to the $\mathrm{NIH}$ criteria in seven studies (Guzick et al. 1994, Moran et al. 2003, Hoeger et al. 2004, van Dam et al. 2004, Fux Otta et al. 2010, Ladson et al. 2011, Pasquali et al. 2011) and the Rotterdam criteria in 10 studies (Table 1, Column 2). Of the interventions that used the broader definition, three primarily recruited the Frank and Non-PCO phenotypes (Palomba et al. 2008, 2010, Nybacka et al. 2011). The inclusion of these two phenotypes implies that the majority of participants had the severest manifestations of the condition and met both the NIH and Rotterdam criteria for PCOS. The remaining studies $(n=7)$ evaluated ovulatory response across more heterogeneous cohorts. Of these, four enrolled participants who could be stratified into any of the recognized phenotypes, and as such, included women with evidence of regular ovulation and menstrual cycles at baseline (Moran et al. 2006, 2007b, Qublan et al. 2007, Thomson et al. 2008). In some cases, these studies presented outcome data on ovulation for the entire cohort and did not distinguish the women with histories of anovulation from those with normal ovulatory function prior to the intervention (Moran et al. 2006, 2007b) (Table 1, Column 2). The combined assessment of ovulatory status in these distinct cohorts may have masked the independent effect of dietary intervention on ovulation as improvements would have been challenging to characterize in women with existing ovulatory cyclicity. The other three studies enrolled anovulatory women irrespective of androgen status (Crosignani et al. 2003, Thomson et al. 2009, Kuchenbecker et al. 2011). Crosignani and coworkers required participants to demonstrate combined evidence of chronic anovulation or amenorrhea and polycystic ovarian morphology (Crosignani et al. 2003). The absence of inclusion criteria or data to corroborate androgen excess suggested that the cohort largely 
comprised women with the normoandrogenic phenotype (Crosignani et al. 2003). By contrast, Thomson and coworkers and Kuchenbecker and coworkers screened for hyperandrogenism and the resulting cohorts appeared to include all variants except ovulatory PCOS (Thomson et al. 2009, Kuchenbecker et al. 2011) (Table 1, Column 2). It is possible that weight loss would have differential effects on ovarian androgen production in normoandrogenic compared to hyperandrogenic phenotypes and that such differences would be difficult to capture using these definitions.

\section{Degree of change in salient endocrine or metabolic features afforded by the intervention}

The dichotomization of ovulatory response to weight loss may also reflect the ability of hypocaloric dietary intervention to recover the endocrine and metabolic abnormalities that impair antral follicle development. Currently, little is known about the salient features or degree of change required to improve reproductive outcomes with weight loss in PCOS.

Ten studies evaluated differences in clinical, endocrine and metabolic characteristics between responders and non-responders at the end of the dietary intervention (Hoeger et al. 2004, van Dam et al. 2004, Moran et al. 2007a, Palomba et al. 2008, Thomson et al. 2008, 2009, Kuchenbecker et al. 2011, Nybacka et al. 2011, Pasquali et al. 2011) (Table 4). Compared to non-responders, women with ovulatory or menstrual improvements after weight loss demonstrated greater reductions in weight or BMI, central adiposity, hirsutism or hyperandrogenemia and indices of insulin resistance (Moran et al. 2003, 2007a, Hoeger et al. 2004, van Dam et al. 2004, Palomba et al. 2008, Thomson et al. 2008, 2009, Kuchenbecker et al. 2011, Nybacka et al. 2011, Pasquali et al. 2011). Changes in gonadotropins and ovarian hormones were similar between groups (van Dam et al. 2004, Palomba et al. 2008, Thomson et al. 2008, 2009, Pasquali et al. 2011) (Table 4). Hence, the body of evidence suggests that improvements in weight, adiposity, androgens and insulin are the primary mediators of restored ovulatory function after hypocaloric dietary intervention in PCOS (Fig. 1).
Nevertheless, it is prudent to consider that these analyses produced inconsistent results across studies. Although the majority of authors noted greater changes among responders, a substantial portion (37-40\%) were unable to identify significant differences in weight, hyperandrogenism or insulin sensitivity between groups (van Dam et al. 2004, Moran et al. 2007a, Thomson et al. 2008, 2009) (Table 4). These findings could reflect heterogeneity in the study populations (as described previously) or a larger issue surrounding the efficacy of the interventions that were used.

The primary therapeutic targets of caloric restriction are weight and adiposity (Jensen et al. 2014). In PCOS, it is likely that changes in weight and adiposity stimulate improvements in androgens and insulin, which together precede ovulation and menses (Moran et al. 2006). If sufficient weight loss is not achieved, then it follows that improvements in ovulatory function would be unlikely to occur (Fig. 1). In the studies included for review, women experienced sporadic ovulation with modest changes in weight (i.e. $<16 \%$ ) (Tables 1 and 2). This occurred despite the fact that most women were still classified as obese (BMI $\geq 30 \mathrm{~kg} / \mathrm{m}^{2}$ ) at the end of the various dietary interventions (Hoeger et al. 2004, van Dam et al. 2004, Fux Otta et al. 2010, Kuchenbecker et al. 2011, Nybacka et al. 2011). A greater degree of weight loss, resulting in a healthier $\mathrm{BMI}$, may be necessary to fully restore ovulatory cyclicity in obese women with PCOS. This idea is supported by preliminary evidence from studies involving bariatric surgery, wherein $100 \%$ of anovulatory patients resumed regular ovulation and menses after a $41-\mathrm{kg}$ reduction in weight (EscobarMorreale et al. 2005). However, surgical approaches for weight loss have been associated with significant risks, including post-operative complications, nutritional deficiencies and increased likelihood for deleterious fetal outcomes during pregnancy (Moran \& Norman 2012). Therefore, it is considered wisest to first advocate for dietary and lifestyle modifications in obese women with PCOS (Moran \& Norman 2012). Further studies are needed to determine the optimal degree of weight loss to induce and sustain improvements in ovulatory function, so as to better tailor dietary interventions to individual needs.

Table 4 Studies that have linked changes in clinical, endocrine and metabolic characteristics to improved reproductive outcomes after hypocaloric dietary intervention in overweight or obese women with PCOS.

\begin{tabular}{|c|c|c|}
\hline Outcome & $\begin{array}{l}\text { Number of studies that found } \\
\text { greater changes in responders }\end{array}$ & $\begin{array}{l}\text { Number of studies that found } \\
\text { no differences in responders }{ }^{a}\end{array}$ \\
\hline Decrease in weight or BMI & $5 / 8(63 \%)$ & $3 / 8(37 \%)$ \\
\hline Decrease in central adiposity & $5 / 6(83 \%)$ & $1 / 6(17 \%)$ \\
\hline Decrease in hirsutism or hyperandrogenemia & $3 / 5(60 \%)$ & $2 / 5(40 \%)$ \\
\hline Improvement in insulin sensitivity & $5 / 8(63 \%)$ & $3 / 8(37 \%)$ \\
\hline Decrease in mean concentrations of gonadotropins or ovarian hormones ${ }^{b}$ & $1 / 4(25 \%)$ & $3 / 4(75 \%)$ \\
\hline
\end{tabular}

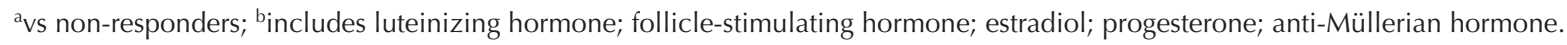


In addition, little is known about the time course of caloric restriction that is required to improve ovarian function in obese women with PCOS. Although serial assessments of ovulatory status were performed, the majority of studies did not report the time point(s) of the intervention at which ovulation was observed. Only three studies provided information on this outcome. From these data, it appeared unlikely for an ovarian response to occur within the first two weeks of a dietary intervention (Moran et al. 2003, van Dam et al. 2004, Palomba et al. 2010). Specifically, Moran and coworkers did not detect sporadic ovulation until weeks $4-6$ or 12-13 of caloric restriction (Moran et al. 2003). The time course of these observations may be explained by evidence that endocrine and metabolic responses to caloric restriction precede ovulation (van Dam et al. 2004, Moran et al. 2006). Alterations in neuroendocrine feedback were identified in as a few as seven days on a very low-calorie diet (van Dam et al. 2004), and significant improvements in androgen excess and insulin sensitivity were documented within 2-4 weeks after the onset of dietary intervention (Moran et al. 2006). Consequently, a decrease in insulin-mediated androgen production (Barbieri et al. 1986) might be expected to stimulate sporadic selection and ovulation several weeks later (Moran et al. 2006).

Despite the occurrence of sporadic ovulation, it remains unclear whether short-term caloric restriction is sufficient to normalize ovulatory cyclicity in obese women with PCOS. It has been suggested that turnover of the antral follicles recruited under hyperandrogenic and insulin-resistant conditions precedes improvements in ovarian function (Thomson et al. 2009). Changes in the mechanisms of selection and ovulation may not manifest until a new cohort of follicles is activated from the primordial pool under an improved hormonal environment. Given that a pre-antral follicle takes approximately three months to reach its pre-ovulatory diameter (Gougeon 1986), it is possible that some of the dietary interventions were too short to realize improvements in reproductive function (Thomson et al. 2009) (Table 1). Further investigation is needed to determine the precise time course of caloric restriction that is required to normalize ovulatory cyclicity in PCOS.

It is also uncertain whether improvements in ovulatory function can be sustained after a hypocaloric dietary intervention is discontinued. In general, weight maintenance is challenging and weight re-gain is a common problem across populations (MacLean et al. 2014). This is essential to address in the context of PCOS, as any increase in weight could recover initial endocrine and metabolic disturbances and lead to the rebound of anovulation. Five studies performed follow-up assessments and attempted to capture these changes after the dietary intervention (Moran et al. 2003, 2006, Hoeger et al. 2004, Nybacka et al. 2011, Pasquali et al. 2011). Follow-up assessments were performed on a cross-sectional basis (Nybacka et al. 2011, Pasquali et al. 2011) or as part of a weight maintenance intervention that involved regular visits to the research unit (Moran et al. 2003, 2006, Hoeger et al. 2004). Ovulatory or menstrual status was largely measured at each time point (Moran et al. 2003, 2006, Hoeger et al. 2004, Nybacka et al. 2011). Yet, only two of the studies distinguished the data that were collected at the follow-up visits from those collected during the intervention, and menstrual status was the primary reproductive outcome of interest (Moran et al. 2003, Nybacka et al. 2011). Specifically, Moran and coworkers found that improvements in menstrual cyclicity were sustained for 4-6 months after caloric restriction in the majority of patients (Moran et al. 2003). In some cases, women reported regular menses for an average of two years after the end of the intervention (Nybacka et al. 2011). These outcomes occurred independent of increased energy intake (Moran et al. 2003) or weight gain (Moran et al. 2003, Nybacka et al. 2011). Further studies are needed to clarify whether and how these sustained changes in menstrual cyclicity are reflected in the ovaries.

Despite ambiguity surrounding the ideal dietary intervention, current dogma stipulates that caloric restriction is the primary facilitator of weight loss and ovulation in obese women with PCOS (Moran et al. 2009, 2013). Consequently, dietary interventions involving caloric restriction were highlighted in this review. However, it is important to acknowledge that most of the included studies prescribed caloric restriction as part of a larger multifactorial intervention (Table 1, Column 5). Emerging data suggest that tailored macronutrient composition, physical activity and/or behavior modification therapy can augment the effect of caloric restriction on ovulatory function (Harrison et al. 2011, Moran et al. 2013). Although these additional approaches were not addressed in this review, they have been described extensively by Moran and coworkers (Moran et al. 2013) and Harrison and coworkers (Harrison et al. 2011). Ultimately, welldesigned, randomized controlled trials are still required to determine whether multifactorial dietary interventions can further improve reproductive outcomes in PCOS (Harrison et al. 2011, Moran et al. 2013).

\section{Conclusion}

In summary, commendable progress has been made toward understanding the impact of caloric restriction on ovulatory function in obese women with PCOS. It is clear that modest weight loss is associated with the occurrence of sporadic ovulation in a meaningful proportion of patients and that reductions in hyperandrogenism and insulin resistance likely precede any improvements in reproductive outcomes. The ovulatory response may also depend on the presence of milder reproductive 
dysfunction at baseline and a greater degree of change in endocrine and metabolic features with intervention. Nevertheless, this review highlighted the variability in the ovulatory response to weight loss and found little evidence to support the effectiveness of hypocaloric dietary intervention to restore normal ovulatory function in PCOS. Because resumption of regular ovulatory cycles may not be a realistic goal for all patients, health care providers should be judicious in counseling the degree to which weight loss can be expected to improve ovulatory function in obese patients with PCOS. Future studies would benefit from efforts to improve the accuracy and consistency of measures used to determine and report ovulatory status both at baseline and during dietary intervention. The use of intermittent sampling of biochemical markers, assessment of alternative thresholds for ovulation to better judge luteal function in PCOS and distinction of ovulation from menstrual cyclicity in reporting improvements is recommended. Finally, the impact of phenotypic variation on the ovulatory response to weight loss should be addressed to fully capture the degree and duration of caloric restriction that is needed to effectively promote weight loss and ovulation in PCOS. The identification of optimal dietary and lifestyle approaches to treat anovulation will improve the health and wellbeing of obese women living with PCOS.

\section{Declaration of interest}

The authors declare that there is no conflict of interest that could be perceived as prejudicing the impartiality of the review reported.

\section{Funding}

This work was supported by the Division of Nutritional Sciences at Cornell University and the National Institutes of Health (T32-DK007158). The context is solely the responsibility of the authors and does not necessarily represent the official views of the National Institute of Diabetes and Digestive Kidney Diseases (NIDDK) or the National Institutes of Health.

\section{Acknowledgements}

The authors would like to thank Annie W Lin for her insight and assistance in the preparation of this manuscript.

\section{References}

Baerwald A, Adams G \& Pierson R 2012 Ovarian antral folliculogenesis during the human menstrual cycle: a review. Human Reproduction Update 18 73-91. (doi:10.1093/humupd/dmr039)

Barbieri R, Makris A, Randall R, Daniles G, Kistner R \& Ryan K 1986 Insulin stimulates androgen accumulation in incubations of ovarian stroma obtained from women with hyperandrogenism. Journal of Clinical Endocrinology and Metabolism 62 904-910. (doi:10.1210/ jcem-62-5-904)
Bützow T, Lehtovirta M, Siegberg R, Hovatta O, Koistinen R, Seppälä M \& Apter D 2000 The decrease in luteinizing hormone secretion in response to weight reduction is inversely related to the severity of insulin resistance in overweight women. Journal of Clinical Endocrinology and Metabolism 85 3271-3275. (doi:10.1210/jc.85.9.3271)

Campbell K \& Rockett J 2006 Biomarkers of ovulation, endometrial receptivity, fertilisation, implantation and early pregnancy progression. Paediatric and Perinatal Epidemiology 20 13-25. (doi:10.1111/j.13653016.2006.00766.x)

Carmina E, Chu M, Longo R, Rini G \& Lobo R 2005 Phenotypic variation in hyperandrogenic women influences the findings of abnormal metabolic and cardiovascular risk parameters. Journal of Clinical Endocrinology and Metabolism 90 2545-2549. (doi:10.1210/jc.2004-2279)

Creinin M, Keverline S \& Meyn L 2004 How regular is regular? An analysis of menstrual cycle regularity. Contraception 70 289-292. (doi:10.1016/j. contraception.2004.04.012)

Crosignani P, Colombo M, Vegetti W, Somigliana E, Gessati A \& Ragni G 2003 Overweight and obese anovulatory patients with polycystic ovaries: parallel improvements in anthropometric indices, ovarian physiology and fertility rate induced by diet. Human Reproduction $\mathbf{1 8}$ 1928-1932. (doi:10.1093/humrep/deg367)

van Dam E, Roelfsema F, Veldhuis J, Hogendoorn S, Westenberg J, Helmerhorst F, Frölich M, Krans H, Meinders A \& Pijl H 2004 Retention of estradiol negative feedback relationship to $\mathrm{LH}$ predicts ovulation in response to caloric restriction and weight loss in obese patients with polycystic ovary syndrome. American Journal of Physiology Endocrinology and Metabolism 286 E615-E620. (doi:10.1152/ ajpendo.00377.2003)

Dewailly D, Catteau-Jonard S, Reyss A-C, Leroy M \& Pigny P 2006 Oligoanovulation with polycystic ovaries but not overt hyperandrogenism. Journal of Clinical Endocrinology and Metabolism 91 3922-3927. (doi:10.1210/jc.2006-1054)

Diamanti-Kandarakis E \& Dunaif A 2012 Insulin resistance and the polycystic ovary syndrome revisited: an update on mechanisms and implications. Endocrine Reviews 33 981-1030. (doi:10.1210/ er.2011-1034)

Escobar-Morreale H, Botella-Carretero J, Álvarez-Blasco F, Sancho J \& San Millán J 2005 The polycystic ovary syndrome associated with morbid obesity may resolve after weight loss induced by bariatric surgery. Journal of Clinical Endocrinology and Metabolism 90 6364-6369. (doi:10.1210/ jc.2005-1490)

Fauser B, Tarlatzis B, Rebar R, Legro R, Balen A, Lobo R, Carmina E, Chang J, Yildiz B, Laven J et al. 2012 Consensus on women's health aspects of polycystic ovary syndrome (PCOS): the Amsterdam ESHRE/ASRM-Sponsored 3rd PCOS Consensus Workshop Group. Fertility and Sterility 97 28-38. (doi:10.1016/j. fertnstert.2011.09.024)

Franks S, Stark J \& Hardy K 2008 Follicle dynamics and anovulation in polycystic ovary syndrome. Human Reproduction Update 14 367-278. (doi:10.1093/humupd/dmn015)

Fux Otta C, Wior M, Iraci G, Kaplan R, Torres D, Gaido M \& Wyse E 2010 Clinical, metabolic, and endocrine parameters in response to metformin and lifestyle intervention in women with polycystic ovary syndrome: a randomized, double-blind, and placebo control trial. Gynecological Endocrinology 26 173-178. (doi:10.3109/09513590903215581)

Gougeon A 1986 Dynamics of follicular growth in the human: a model from preliminary results. Human Reproduction 1 81-87.

Guzick D, Wing R, Smith D, Berga S \& Winters S 1994 Endocrine consequences of weight loss in obese, hyperandrogenic, anovulatory women. Fertility and Sterility 61 598-604. (doi:10.1016/S00150282(16)56632-1)

Harrison C, Lombard C, Moran L \& Teede H 2011 Exercise therapy in polycystic ovary syndrome: a systematic review. Human Reproduction Update 17 171-183. (doi:10.1093/humupd/dmq045)

Hoeger K, Kochman L, Wixom N, Craig K, Miller R \& Guzick D 2004 A randomized, 48-week, placebo-controlled trial of intensive lifestyle modification and/or metformin therapy in overweight women with polycystic ovary syndrome: a pilot study. Fertility and Sterility $\mathbf{8 2}$ 421-429. (doi:10.1016/j.fertnstert.2004.02.104)

Institute of Medicine 2005 Dietary References Intakes for Energy, Carbohydrate, Fiber, Fat, Fatty Acids, Cholesterol, Protein, and Amino 
Acids (Macronutrients). Washington, DC, USA: The National Academies Press. (doi:10.17226/10490)

Jensen M, Ryan D, Donato K, Apovian C, Ard J, Comuzzie A, Hu F, Hubbard V, Jakicic J, Kushner R et al. 2014 Guidelines (2013) for managing overweight and obesity in adults. Obesity 22 S1-S410. (doi:10.1002/oby.20660)

Jonard S \& Dewailly D 2004 The follicular excess in polycystic ovaries, due to intraovarian hyperandrogenism, may be the main culprit for the follicular arrest. Human Reproduction Update 10 107-117. (doi:10.1093/humupd/dmh010)

Joseph-Horne R, Mason H, Batty S, White D, Hillier S, Urquhart M \& Franks S 2002 Luteal phase progesterone excretion in ovulatory women with polycystic ovaries. Human Reproduction 17 1459-1463. (doi:10.1093/humrep/17.6.1459)

Kuchenbecker W, Groen H, van Asselt S, Bolster J, Zwerver J, Slart R, Vd Jagt E, Muller Kobold A, Wolffenbuttel B, Land J et al. 2011 In women with polycystic ovary syndrome and obesity, loss of intraabdominal fat is associated with resumption of ovulation. Human Reproduction 26 2505-2512. (doi:10.1093/humrep/der229)

Ladson G, Dodson W, Sweet S, Archibong A, Kunselman A, Demers L, Williams N, Coney P \& Legro R 2011 The effects of metformin with lifestyle therapy in polycystic ovary syndrome: a randomized doubleblind study. Fertility and Sterility 95 1059-1066. (doi:10.1016/j. fertnstert.2010.12.002)

Legro R, Arslanian S, Ehrmann D, Hoeger K, Murad M, Pasquali R \& Welt C 2013 Diagnosis and treatment of polycystic ovary syndrome: an endocrine society clinical practice guideline. Journal of Clinical Endocrinology and Metabolism 98 4565-4592. (doi:10.1210/ jc.2013-2350)

Leiva R, Bouchard T, Boehringer H, Abulla S \& Ecochard R 2015 Random serum progesterone threshold to confirm ovulation. Steroids 101 125-129. (doi:10.1016/j.steroids.2015.06.013)

Lenton E, Weston G \& Cooke I 1977 Problems in using basal body temperature recordings in an infertility clinic. BMJ $\mathbf{1}$ 803-805. (doi:10.1136/bmj.1.6064.803)

Lim S, Norman R, Davies M \& Moran L 2013 The effect of obesity on polycystic ovary syndrome: a systematic review and meta-analysis. Obesity Reviews 14 95-109. (doi:10.1111/j.1467789X.2012.01053.x)

Lynch C, Jackson L \& Louis G 2006 Estimation of the day-specific probabilities of conception, current state of the knowledge and the relevance for epidemiological research. Paediatric and Perinatal Epidemiology 20 3-12. (doi:10.1111/j.1365-3016.2006.00765.x)

MacLean P, Wing R, Davidson T, Epstein L, Goodpaster B, Hall K, Levin B, Perri M, Rolls B, Rosenbaum M et al. $2014 \mathrm{NIH}$ working group report: innovative research to improve maintenance of weight loss. Obesity $\mathbf{2 3}$ 7-15. (doi:10.1002/oby.20967)

Moran L \& Norman R 2012 The effect of bariatric surgery on female reproductive function. Journal of Clinical Endocrinology and Metabolism 97 4352-4354. (doi:10.1210/jc.2012-3606)

Moran L \& Teede H 2009 Metabolic features of the reproductive phenotypes of polycystic ovary syndrome. Human Reproduction Update $\mathbf{1 5}$ 477-488. (doi:10.1093/humupd/dmp008)

Moran L, Noakes M, Clifton P, Tomlinson L \& Norman R 2003 Dietary composition in restoring reproductive and metabolic physiology in overweight women with polycystic ovary syndrome. Journal of Clinical Endocrinology and Metabolism 88 812-819. (doi:10.1210/jc.2002020815)

Moran L, Noakes M, Clifton P, Wittert G, Williams G \& Norman R 2006 Short-term meal replacements followed by dietary macronutrient restriction enhance weight loss in polycystic ovary syndrome. American Journal of Clinical Nutrition 84 77-87.

Moran L, Noakes M, Clifton P \& Norman R 2007a The use of antimullerian hormone in predicting menstrual response after weight loss in overweight women with polycystic ovary syndrome. Journal of Clinical Endocrinology and Metabolism 92 3796-3802. (doi:10.1210/ jc.2007-1188)

Moran L, Noakes M, Clifton P, Wittert G, Belobrajdic D \& Norman R $2007 b$ C-reactive protein before and after weight loss in overweight women with and without polycystic ovary syndrome. Journal of Clinical Endocrinology and Metabolism 92 2944-2951. (doi:10.1210/ jc.2006-2336)
Moran L, Pasquali R, Teede H, Hoeger K \& Norman R 2009 Treatment of obesity in polycystic ovary syndrome: a position statement of the Androgen Excess and Polycystic Ovary Syndrome Society. Fertility and Sterility 92 1966-1982. (doi:10.1016/j. fertnstert.2008.09.018)

Moran L, Hutchison S, Norman R \& Teede H 2011 Lifestyle changes in women with polycystic ovary syndrome. Cochrane Database of Systematic Reviews, Issue 7. Art. No: CD007506. (doi:10.1002/14651858. CD007506.pub3)

Moran L, Ko H, Misso M, Marsh K, Noakes M, Talbot M, Frearson M, Thondan M, Stepto N \& Teede H 2013 Dietary composition in the treatment of polycystic ovary syndrome: a systematic review to inform evidence-based guidelines. Journal of the Academy of Nutrition and Dietetics 113 520-545. (doi:10.1016/j.jand.2012.11.018)

Nybacka A, Carlström K, Ståhle A, Nyrén S, Hellström P \& Hirschberg A 2011 Randomized comparison of the influence of dietary management and/or physical exercise on ovarian function and metabolic parameters in overweight women with polycystic ovary syndrome. Fertility and Sterility 96 1508-1513. (doi:10.1016/j. fertnstert.2011.09.006)

Nybacka A, Carlström K, Fabri F, Hellström P \& Hirschberg A 2013 Serum anti-mullerian hormone in response to dietary management and/or physical exercise in overweight/obese women with polycystic ovary syndrome: secondary analysis of a randomized controlled trial. Fertility and Sterility $\mathbf{1 0 0}$ 1096-1102. (doi:10.1016/j. fertnstert.2013.06.030)

O'Connor K, Brindle E, Miller R, Shofer J, Ferrell R, Klein N, Soules M, Holman D, Mansfield P \& Wood J 2006 Ovulation detection methods for urinary hormones: precision, daily and intermittent sampling and a combined hierarchical method. Human Reproduction 21 1442-1452. (doi:10.1093/humrep/dei497)

Palomba S, Giallauria F, Falbo A, Russo T, Oppedisano R, Tolino A, Colao A, Vigorito C, Zullo F \& Orio F 2008 Structured exercise training programme versus hypocaloric hyperproteic diet in obese polycystic ovary syndrome patients with anovulatory infertility: a 24-week pilot study. Human Reproduction 23 642-650. (doi:10.1093/humrep/ dem391)

Palomba S, Falbo A, Giallauria F, Russo T, Rocca M, Tolino A, Zullo F \& Orio F 2010 Six weeks of structured exercise training and hypocaloric diet increases the probability of ovulation after clomiphene citrate in overweight and obese patients with polycystic ovary syndrome: a randomized controlled trial. Human Reproduction 25 2783-2791. (doi:10.1093/humrep/deq254)

Panidis D, Tziomalos K, Misichronis G, Papadakis E, Betsas G, Katsikis I \& Macut D 2012 Insulin resistance and endocrine characteristics of the different phenotypes of polycystic ovary syndrome: a prospective study. Human Reproduction 27 541-549. (doi:10.1093/humrep/ der418)

Pasquali R, Gambineri A, Cavazza C, Gasparini D, Ciampaglia W, Cognigni G \& Pagotto U 2011 Heterogeneity in the responsiveness to long-term lifestyle intervention and predictability in obese women with polycystic ovary syndrome. European Journal of Endocrinology 164 53-60. (doi:10.1530/EJE-10-0692)

Prior J, Naess M, Langhammer A \& Forsmo S 2015 Ovulation prevalence in women with spontaneous normal-length menstrual cycles - a population-based cohort from HUNT3, Norway. PLOS ONE $\mathbf{1 0}$ e0134473. (doi:10.1371/journal.pone.0134473)

Qublan H, Yannakoula E, Al-Qudah M \& El-Uri F 2007 Dietary intervention versus metformin to improve the reproductive outcome in women with polycystic ovary syndrome. Saudi Medical Journal 28 1694-1699.

Rochester D, Jain A, Polotsky A, Polotsky H, Gibbs K, Isaac B, Zeitlian G, Hickmon C, Feng S \& Santoro N 2009 Partial recovery of luteal function after bariatric surgery in obese women. Fertility and Sterility $\mathbf{9 2}$ 1410-1415. (doi:10.1016/j.fertnstert.2008.08.025)

Rojas J, Chávez M, Olivar L, Milagros R, Morillo J, Mejías J, Calvo M \& Bermúdez V 2014 Polycystic ovary syndrome, insulin resistance, and obesity: navigating the pathophysiologic labyrinth. International Journal of Reproductive Medicine 2014719050. (doi:10.1155/2014/719050)

Roos J, Johnson S, Weddell S, Godehardt E, Schiffner J, Freundl G \& Gnoth C 2015 Monitoring the menstrual cycle: comparison of urinary and serum reproductive hormones referenced to true ovulation. 
European Journal of Contraception and Reproductive Health Care 20 438-450. (doi:10.3109/13625187.2015.1048331)

Rotterdam ESHRE/ASRM-Sponsored PCOS Consensus Workshop Grouep 2004a Revised 2003 consensus on diagnostic criteria and long-term health risks related to polycystic ovary syndrome. Fertility and Sterility 81 19-25. (doi:10.1016/j.fertnstert.2003.10.004)

Rotterdam ESHRE/ASRM-Sponsored PCOS Consensus Workshop Group $2004 b$ Revised 2003 consensus on diagnostic criteria and long-term health risks related to polycystic ovary syndrome (PCOS). Human Reproduction 19 41-47. (doi:10.1093/humrep/deh098)

Thomson R, Buckley J, Noakes M, Clifton P, Norman R \& Brinkworth G 2008 The effect of a hypocaloric diet with and without exercise training on body composition, cardiometabolic risk profile, and reproductive function in overweight and obese women with polycystic ovary syndrome. Journal of Clinical Endocrinology and Metabolism 93 3373-3380. (doi:10.1210/jc.2008-0751)

Thomson R, Buckley J, Moran L, Noakes M, Clifton P, Norman R \& Brinkworth G 2009 The effect of weight loss on anti-Müllerian hormone levels in overweight and obese women with polycystic ovary syndrome and reproductive impairment. Human Reproduction 24 1976-1981. (doi:10.1093/humrep/dep101)

Wathen N, Perry L \& Chard T 1984 Interpretation of single progesterone measurement in diagnosis of anovulation and defective luteal phase: observations on analysis of the normal range. British Medical Journal 288 7-9. (doi:10.1136/bmj.288.6410.7)

Zawadzki J \& Dunaif A 1992 Diagnostic criteria for polycystic ovary syndrome: towards a rational approach. In Polycystic Ovary Syndrome, pp 377-384. Eds A Dunaif, J Givens \& F Haseltine. Boston, USA: Blackwell Scientific.

Received 15 July 2016

First decision 24 August 2016

Revised manuscript received 6 October 2016

Accepted 26 October 2016 